\title{
Nouvelles perspectives de diagnostics de plasmas denses par génération d'harmoniques d'ordre élevé
}

\author{
H. Merdji, P. Salières, J.-F. Hergott, P. Monot, P. d'Oliveira, T. Auguste, B. Carré, \\ D. Descamps ${ }^{1}$, C. Lyngå1 ${ }^{1}$ J. Norin ${ }^{1}$, A. L'Huillier ${ }^{1}$, C.-G. Wahlström ${ }^{1}$, M. Bellini', \\ T.W. Hänsch ${ }^{3}$ et S. Hüller ${ }^{4}$
}

\author{
Service des Photons, Atomes et Molécules, CEA-DSM-DRECAM, Centre de Saclay, \\ 91191 Gif-sur-Yvette, France \\ ${ }^{1}$ Department of Physics, Lund Institute of Technology, P.O. Box 118, 22100 Lund, Suède \\ 2 LENS, Largo E. Fermi 2, 50125 Florence, Italie \\ ${ }^{3}$ Max-Planck-Institut für Quantenoptik, P.O. Box 1513, 85740 Garching, Allemagne \\ ${ }^{4}$ Centre de Physique Théorique, CNRS, École Polytechnique, 91128 Palaiseau cedex, France
}

\begin{abstract}
Résumé : Les propriétés de cohérence ainsi que la très courte durée des impulsions de la génération d'harmonique d'ordre élevé ont été appliquées à des applications de type pompe/sonde utilisant l'interférométrie dans le domaine XUV. Nous avons pour la première fois mesuré dans deux expériences différentes les densités électroniques de plasmas par interférométrie fréquentielle et spatiale dans le domaine XUV avec une résolution temporelle de quelques dizaine de femtosecondes.
\end{abstract}

\section{INTRODUCTION}

Les plasmas sont étudiés dans de nombreuses disciplines de la physique (fusion par confinement inertiel, astrophysique, sources $\mathrm{X}$ incohérentes, lasers XUV, ...). L'interférométrie infra-rouge (IR) est largement utilisée pour mesurer les densités électroniques de tels plasmas. Cependant cette technique reste limitée aux régions de faible densité (au mieux quelques $10^{20} \mathrm{~cm}^{-3}$ ) notamment en raison de l'absorption et de la réfraction du rayonnement par le plasma. L'étude de plasmas denses sur-critiques $\mathrm{N}_{\text {crit. }}=10^{21} \mathrm{~cm}^{-3}$ pour l'IR- doit nécessairement recourir à l'interférométrie XUV. L'extension de cette technique au domaine XUV a longtemps posé problème par manque de sources XUV suffisamment brillantes et d'éléments optiques de haute qualité. Ce n'est que récemment, avec le synchrotron puis le laser $\mathrm{X}$, que des diagnostics interférométriques ont pu être réalisés, prolongeant le champ d'application de cette technique à des milieux opaques à la lumière visible. Le nombre d'installations lourdes possédant les propriétés nécessaires étant limité, les études menées à ce jour restent peu nombreuses. Elles concernent la caractérisation structurale de surfaces ou films solides et le diagnostic de densités électroniques dans des plasmas, en régime stationnaire, $n$ 'incluant pas ou peu les aspects dynamiques qui restent limités à l'échelle de la nanoseconde par la durée des impulsions. Pourtant, dans de nombreux domaines tels que la physique du solide ou des plasmas, les études de phénomènes ultra-rapides offrent un large champ d'applications à l'interférométrie XUV.

La génération, dans un gaz, des harmoniques d'ordre élevé d'un champ laser fondamental se distingue notoirement des autres sources comme le rayonnement synchrotron ou l'émission de plasmas. Il ne s'agit pas en effet de source à proprement parler, mais de rayonnement converti en fréquence. Du caractère cohérent du processus de conversion, il découle des caractéristiques uniques de durée, de cohérence spatiale et temporelle, de divergence qui reflètent celles du champ laser fondamental. Nous présentons ici deux expériences d'interférométrie utilisant la génération d'harmoniques d'ordre élevé. Dans la première expérience d'application, nous avons utilisé la possibilité de produire deux sources harmoniques séparées spatialement et bloquées en phase (donc mutuellement cohérentes) [1]. De la 
même manière que dans l'expérience des fentes d'Young ces deux sources donnent lieu à des figures d'interférence en champ lointain. Nous avons ensuite appliqué ce schéma interférométrique à la mesure de densité d'un plasma d'aluminium [2]. Dans la seconde expérience nous avons montré qu'il était possible de générer deux sources harmoniques séparées en temps et bloquées en phase en focalisant une double impulsion laser au même endroit dans le milieu générateur [3]. Après dispersion du rayonnement par un réseau on observe alors des franges d'interférence dans le domaine des fréquences. Ce schéma a permis d'étudier la dynamique d'ionisation du milieu générateur et nous a donné des informations sur un possible chirp des harmoniques. Cette technique a été utilisée dans une expérience d'interférométrie afin de sonder un plasma dense d'hélium.

\section{INTEROMETRIE SPATIALE DANS LE DOMAINE XUV}

L'expérience a été réalisée au Lund Laser Center, à Lund en Suède. Le schéma est identique à celui utilisé pour caractériser les propriétés de cohérence temporelle du rayonnement harmonique d'ordre élevé [4]. Un laser Ti :Sa délivrant $100 \mathrm{~mJ}$ à $790 \mathrm{~nm}$ en $110 \mathrm{fs}$ à $10 \mathrm{~Hz}$ est séparé en deux bras synchronisés et identiques à l'aide d'un interféromètre de Michelson. Les deux faisceaux sont focalisés dans un jet pulsé de krypton à des intensités pic de l'ordre de $10^{14} \mathrm{~W} / \mathrm{cm}^{2}$ au foyer. Afin de produire deux foyers distincts dans le jet de gaz, un des bras du Michelson est légèrement désaligné. Typiquement, les deux sources harmoniques alors créées sont séparées de $130 \mu \mathrm{m}$. Après propagation, les deux faisceaux se superposent et interfêrent en champ lointain. Le rayonnement harmonique est sélectionné par un réseau sphérique en incidence normale de 1200 lignes $/ \mathrm{mm}$. Les franges sont détectées par des galettes de micro-cannaux couplées à un écran phosphore et digitalisées par une caméra CCD 16 bits.

Nous avons utilisé ce schéma pour sonder un plasma d'aluminium. La possibilité de choisir une longueur d'onde dans le spectre harmonique est crucial afin de se situer dans des conditions telles que la réfraction et l'absorption ne soient pas trop importantes et que le déphasage induit soit suffisamment important pour être mesuré. Le plasma est généré en focalisant une impulsion de $50 \mathrm{~mJ}, 300 \mathrm{ps}$ à $790 \mathrm{~nm}$ sur une feuille d'aluminium et avec un délai de $1.2 \mathrm{~ns}$ avant les deux impulsions harmonique. L'intensité sur cible est de l'ordre de $10^{13} \mathrm{~W} / \mathrm{cm}^{2}$. La cible est placée directement après le jet. Dans ces conditions (faible angle solide et sélection spectrale du réseau), l'émission propre du plasma est en grande partie supprimée.

La longueur d'onde optimale pour sonder le plasma est celle de l'harmonique 11 (113 nm). La figure 1 présente des interférogrammes obtenus en monocoup. Le diagnostic de densité consiste à faire passer un des faisceaux harmoniques (sonde) à travers le plasma alors que le second (référence) se propage sans perturbation à côté du plasma. Le faisceau sonde mesure alors les déphasages induits par les densités électroniques vues dans le plasma. Sur la figure la, la cible n'est pas irradiée et les franges présentent une bonne visibilité. Les figures $1 \mathrm{~b}$ et $1 \mathrm{c}$ ont été obtenues en irradiant la cible $1.2 \mathrm{~ns}$ après la création dub plasma mais à différentes distances cible/sonde, la sonde étant plus proche de la cible sur la figure 1c. Comparé à la figure 1a, le niveau de bruit est plus important à cause de l'émission propre du plasma et les franges d'interférence sont inclinées. Le décalage des franges $N$ à une longueur d'onde $\lambda$ est lié à la densité électronique du plasma $N_{e}$ par la relation:

$$
N=\int_{0}^{d} \frac{1-n_{r e f}}{\lambda} d x \approx \frac{L}{2 \lambda} \frac{N_{e}}{N_{c}},
$$

$n_{\text {réf }}$ et $N_{c}$ étant respectivement l'indice de réfraction du plasma et la densité critique. $d$ est la distance parcourue par le faisceau sonde dans le plasma ; elle a été estimée expérimentalement à $100 \mu \mathrm{m}$. Si l'on considère que la réfraction est négligeable et que le plasma est uniforme sur le chemin optique du faisceau sonde, le décalage de franges est alors directement proportionnel à la densité électronique. Sur la figure $1 \mathrm{~b}$, les franges sont décalées uniquement sur la partie supérieure de l'image. La partie basse de l'image donne donc un système de franges de référence nous permettant d'avoir une mesure absolue de la densité électronique. Des lignes d'isodensité ont été tracées en pointillés correspondant à densités de $0.3,1$ et $2.10^{20}$ électrons $/ \mathrm{cm}^{3}$ (de bas en haut). Sur la figure 1c, le faisceau sonde est totalement perturbé par le plasma de telle sorte que le système de franges entier est incliné. Si l'on utilise la partie basse de l'image comme référence, la position des franges dans le plasma ne donne alors qu'une valeur inférieure de la densité électronique. Nous en déduisons près de la cible des densités d'au moins $2.510^{20}$ électrons $/ \mathrm{cm}^{3}$. Les mesures obtenus permettent de suivre l'expansion 2-D du plasma d'aluminium. Les mesures sont en bon accord avec les simulations réalisées avec le code hydroradiatif MULTI (figure 2). 


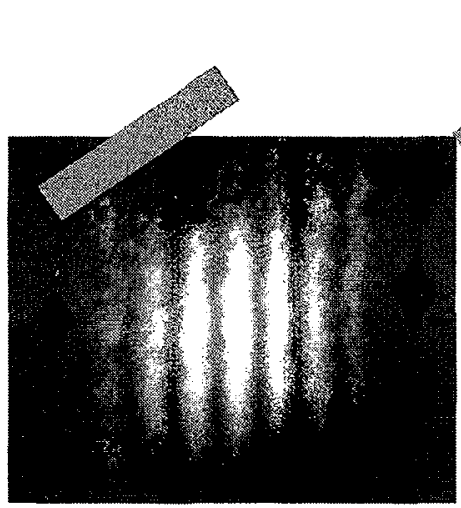

(a)

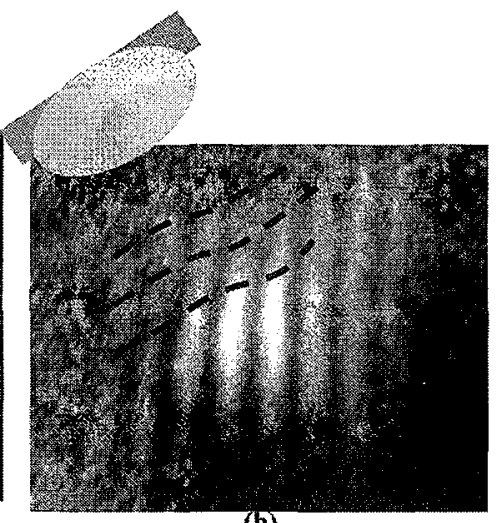

(b)

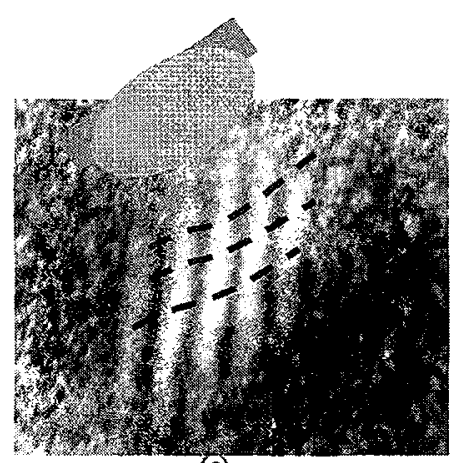

(c)

Figure 1: Interférogrammes obtenus en simple tir avec l'harmonique 11 (113nm): (a) sans plasma, et (b) et (c) avec plasma.

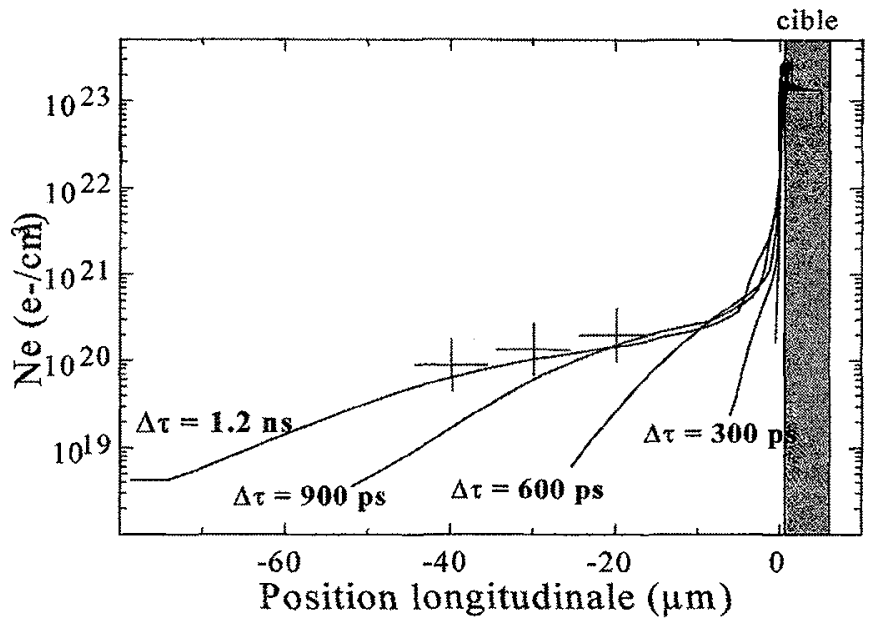

Figure 2: Simulations de l'expansion du plasma à différents délais (traits pleins) et comparaison avec les densités mesurées (croix).

\section{INTEROMETRIE FREQUENTIELLE DANS LE DOMAINE XUV}

L'interférométrie dans le domaine des fréquences peut être introduite en transposant l'expérience classique d'interférométrie des fentes d'Young dans le domaine temporel. Dans le cas de l'interférométrie fréquentielle, deux impulsions séparées dans le temps d'un écart supérieur à leur durée interfêrent dans le domaine des fréquences après dispersion sur un réseau. La transposition de cette techríique à la génération d'harmoniques d'ordre élevé pose deux difficultés majeures. La première prov'ent de la courte longueur d'onde des harmoniques d'ordre élevé. En effet puisque la période des franges en longueur d'onde s'écrit $\Delta \lambda=\lambda^{2} / \mathrm{c} \Delta \mathrm{t}$ celle-ci décroît rapidement avec l'ordre des harmoniques. Cela implique qu'il faut utiliser un spectromètre à haute résolution de façon à pouvoir résoudre les franges à l'intérieur du spectre de l'harmonique considérée. La seconde difficulté provient du processus de génération des harmoniques lui-même. La création de deux sources harmoniques bloquées en phase et séparées temporellement est réalisée en focalisant au même endroit d'un jet de gaz deux impulsions IR décalées temporellement. Or la génération d'harmoniques d'ordre élevé est étroitement corrélée à l'ionisation du milieu, il est en général admis qu'une génération efficace implique un taux d'ionisation élevé puisque l'on est proche de la saturation de l'émission. En conséquence l'ionisation est presque totale à la fín de la première impulsion. Dans ce cas, la deuxième impulsion laser interagit avec un milieu partiellement voir entièrement ionisé ce qui peut réduire significativement l'efficacité de génération, à 
cause principalement de la déplétion du milieu, de la défocalisation du laser fondarnental et du désaccord de phase induit par les électrons libres. Or un déséquilibre d'intensité entre les deux intensité peut conduire à un brouillage important des franges.

L'expérience a été réalisée sur le laser UHI-10 $(800 \mathrm{~nm}, 60 \mathrm{fs} 750 \mathrm{~mJ}, 10 \mathrm{~Hz})$ au CEA Saclay. Deux impulsions IR de durée 60 fs et séparées d'un délai allant de 100 à 450 fs sont focalisées au même endroit dans un jet de xenon. L'allongement temporel (et la sélection spectrale) des deux impulsions harmoniques est réalisé par un réseau plan gravé à 700 traits $/ \mathrm{mm}$. Les figures $3 \mathrm{a}, 3 \mathrm{~b}$ et $3 \mathrm{c}$ montrent les profils spectraux de l'harmonique 15 obtenus avec deux impulsions jumelles séparées de $120 \mathrm{fs}$ et à différentes intensités. La modulation de l'enveloppe spectrale de l'harmonique 15 avec la période attendue montre que les deux sources harmoniques sont bloquées en phase. En mesurant le contraste en fonction de l'intensité on peut notamment de suivre l'ionisation du milieu.

Les spectres de la figure 3 mettent en évidence une décorrélation des parties bleues des spectres, alors que les fréquences rouges restent bloquées en phase et conserve un bon contraste. Le fort accroissement de la densité électronique sur le front montant de la première impulsion est à l'origine de la perte de cohérence. Cet effet montre l'existence d'un chirp négatif des harmoniques, en accord avec des études récentes. Cette asymétrie du spectre est d'autant plus accentuée que la pression statique dans le jet est élevée, et que l'intensité crête à laquelle est soumis le milieu est élevée.

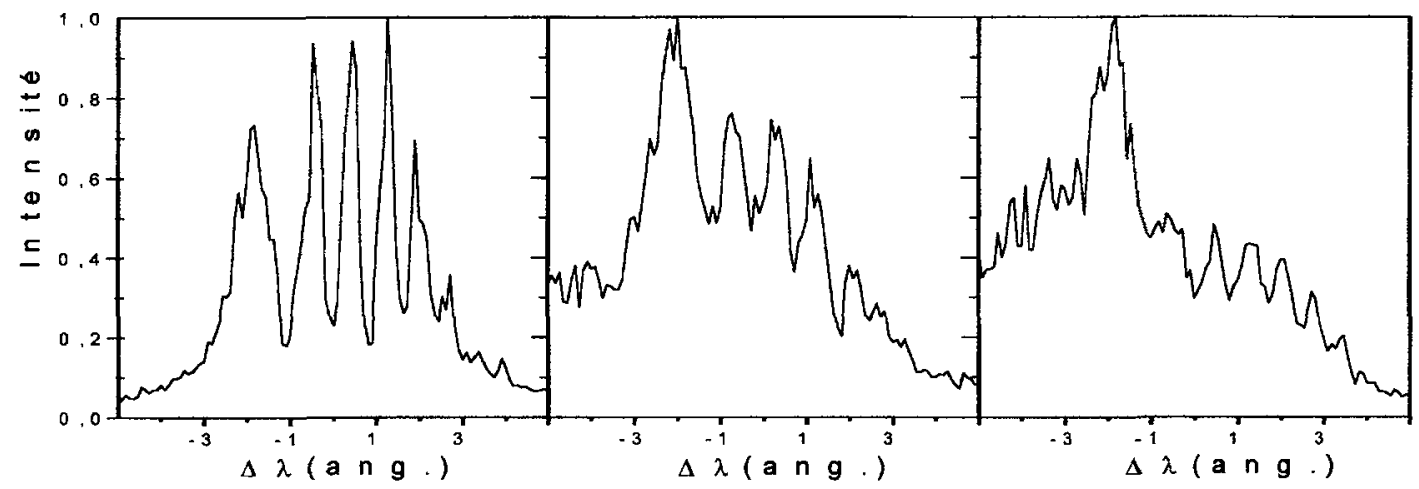

Figure 3: Spectres interférentiels de l'harmonique 15 mesurés pour un délai de $120 \mathrm{fs}$ et à différentes intensités (a): $2.10^{14} \mathrm{~W} / \mathrm{cm}^{2},(\mathrm{~b}): 3,5.10^{14} \mathrm{~W} / \mathrm{cm}^{2}$, (c) $: 5.10^{14} \mathrm{~W} / \mathrm{cm}^{2}$.

Nous avons ensuite mené une expérience d'application à la mesure résolue en temps de la densité électronique d'un plasma d'hélium créé par laser. Une faible fraction de l'énergie du faisceau UHI-10, environ $1 \mathrm{~mJ}$, est prélevée afin de générer l'harmonique 11. Il est à noter que l'ordre de l'harmonique ainsi que le choix du gaz cible sont des paramètres très critiques. Le point délicat est qu'il faut obtenir une densité électronique mesurable dans des conditions où l'harmonique n'est pas absorbée. Les deux impulsions harmonique sont générées dans un jet de xénon avec un retard de $300 \mathrm{fs}$. Elles sont ensuite focalisées sans grandissement par un miroir torique dans un jet d'hélium. Après analyse par le spectromètre XUV, les franges sont détectées par des galettes de microcanaux couplées à un écran phosphore et une caméra CCD. Le plasma est créé en focalisant à f/3.5 à l'aide d'un miroir parabolique, près de $160 \mathrm{~mJ}$ du faisceau principal UHI-10 dans le jet d'hélium haute pression. Une densité atomique de $2.4 \times 10^{19} \mathrm{~cm}^{-3}$ a été mesurée par interférométrie à $800 \mathrm{~nm}$.

Nous avons enregistré de façon systématique, en acquisition monocoup, des réseaux de franges en fonction du délai $t$ entre l'instant de création du plasma et le passage des impulsions harmonique dans le milieu. Le délai est fixé arbitrairement à 0 lorsque le plasma est créé entre les deux sondes. Lorsque le plasma est généré avant $(t>0)$ ou entre les deux impulsions les franges apparaissent décalées. Il est important de noter que la taille du plasma sondé étant supérieure à celle de la tâche harmonique, l'expérience ne présente pas de résolution spatiale. En conséquence le décalage des franges apparaît homogène sur les interférogrammes de la figure 4a. Cette image est obtenue en ajoutant les interférogrammes associés à différents délais (entre $-800 \mathrm{et}+800 \mathrm{fs}$ ). 

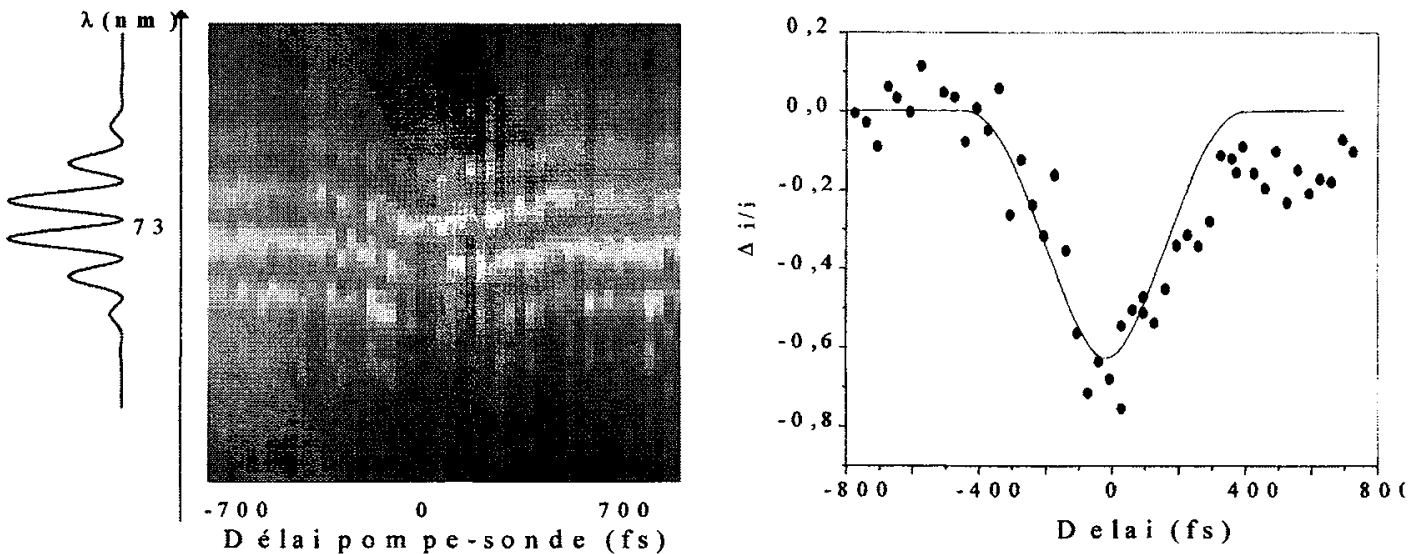

Figure 4: Variation du système de franges (a) et de la position moyenne des franges (b) en fonction du délai entre le laser de pompe et les deux impulsions harmonique 11 .

Un décalage significatif des franges apparaît dans la région centrale de l'image, quand le plasma est créé entre les deux impulsions sondes. Le profil montré sur la figure $4 \mathrm{~b}$ montre le déphasage moyen obtenu à partir de l'image précédente. Après modélisation des paramètres de l'interaction, nous obtenons la courbe en trait plein reportée sur la figure $4 \mathrm{~b}$ qui correspond au décalage de franges théorique obtenu avec les paramètres de l'expérience, en considérant une ionisation instantanée du milieu et un temps de recombinaison très grand devant le délai entre les impulsions sonde. La courbe calculée reproduit bien les variations expérimentales en prenant une densité électronique de $6.10^{19}$ électrons $/ \mathrm{cm}^{3}$. Cette valeur numérique s'accorde assez bien avec la valeur attendue de la densité $\left(4.8 \times 10^{19}\right.$ électrons $\left./ \mathrm{cm}^{3}\right)$ dans le cas de l'ionisation complète des atomes d'hélium. Il est également intéressant de noter qu'un déphasage non nul est mesuré lorsque les deux impulsions harmoniques traversent le plasma. Cet effet a été systématiquement observé et n'est pas encore clairement interprété.

\section{CONCLUSION}

En conclusion, nous avons réalisé les premières applications interférométriques de la génération d'harmonique d'ordre élevé dans les gaz. Nous avons tout d'abord montré que la possibilité de générer simultanément deux sources harmoniques séparées spatialement et bloquées en phase pouvait être utilisée dans une expérience d'interférométrie. Cette technique présente l'avantage de ne nécessiter aucune optique, difficile à produire dans le domaine X-UV. Nous avons pu ainsi réaliser une cartographie 2-D des densités l'ordre de quelques $10^{20}$ électrons $/ \mathrm{cm}^{3} \mathrm{~d}$ 'un plasma d'aluminium crée par laser. La résolution temporelle donnée par la durée du rayonnement harmonique est de l'ordre de $100 \mathrm{fs}$.

Nous avons ensuite montré que la technique d'interférométrie fréquentielle est transposable dans le domaine X-UV en utilisant la génération d'harmonique d'ordre élevé. Il est possible d'obtenir des spectres très réguliers, avec un bon contraste des franges, à condition de limiter l'ionisation du milieu. D'un point de vue fondamental, l'asymétrie des spectres obtenus confirme l'existence d'un chirp négatif du rayonnement harmonique. Enfin, nous avons réalisé la première expérience d'interférométrie fréquentielle dans le domaine XUV. Nous avons ainsi mesuré une densité électronique élevée à l'intérieur d'un plasma généré par l'interaction à haut flux d'un laser et d'une cible d'hélium gazeux.

Ces résultats encourageants montrent qu'il sera possible dans un proche futur de sonder des densités de l'ordre de $10^{23}$ électrons $/ \mathrm{cm}^{3}$ avec des résolutions temporelles de quelques $10 \mathrm{fs}$ et avec une résolution spatiale proche du micron. Dans le contexte de la fusion inertielle par laser, les perspectives se portent notamment sur le concept dit de "l'allumeur rapide». Ces techniques interférométriques ouvrent également d'autres voies d'applications en particulier en physique des surfaces. 


\section{Références}

1. Zerne, R., Altucci C., Bellini M., Gaarde M. B., Hänsch T. W., L'Huillier A., Lynga C., Wahlström C. G., Phys. Rev. Lett., 79, 1006 (1997).

2. Descamps D., Lyngå C., Norin J., L'Huillier A., Wahlström C.-G., Hergott J.-F., Merdji H., Salières P., Bellini M., Hänsch T. W., Optics Letter 25 (2), 135 (2000) et références incluses.

3. Salières, P., Le Déroff, L., Auguste, T., Monot, P., D’Oliveira, P., Campo, D., Hergott, J.-F., Merdji, H., Carré, B., Phys. Rev. Lett. 83, 5483 (1999) et références incluses.

4. Bellini, M. et al., 1998 Phys. Rev. Lett. 81, 297. 